\title{
HispanismeS
}

Revue de la Société des Hispanistes Français

\section{Les poèmes en prose de Federico García Lorca : création d'une disparition}

\section{Carole Fillière}

\section{(2) OpenEdition}

1 Journals

Édition électronique

URL : https://journals.openedition.org/hispanismes/489

DOI : 10.4000/hispanismes.489

ISSN : 2270-0765

Éditeur

Société des Hispanistes Français

Référence électronique

Carole Fillière, "Les poèmes en prose de Federico García Lorca : création d'une disparition », HispanismeS [En ligne], 14 | 2019, mis en ligne le 01 octobre 2019, consulté le 31 août 2021. URL http://journals.openedition.org/hispanismes/489; DOI : https://doi.org/10.4000/hispanismes.489

Ce document a été généré automatiquement le 31 août 2021.

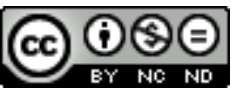

Les contenus de cette revue sont mis à disposition selon les termes de la Licence Creative Commons Attribution - Pas d'Utilisation Commerciale - Pas de Modification 4.0 International. 


\title{
Les poèmes en prose de Federico García Lorca : création d'une disparition
}

\author{
Carole Fillière
}

\section{Introduction}

1 Les Poemas en prosa de Lorca, longtemps relégués dans les marges de son œuvre, sont aujourd'hui identifiés comme une étape créative étroitement associée à des facteurs biographiques: l'amitié et l'émulation artistique entre Lorca et Dalí en 1927 et 1928, alors que le surréalisme fait irruption sur la scène espagnole. Cependant, ces textes ne sont pas des cas isolés dans le processus d'écriture et d'expérimentation de Lorca. En effet, si « la vida de Lorca es un ejercicio ininterrumpido de creación » comme l'affirme Miguel García Posada ${ }^{1}$, il est également possible d'affirmer qu'elle fut un exercice continu d'écriture en prose poétique, comme creuset d'expérimentation pour une évolution, sans limites génériques arbitrairement imposées par des canons littéraires ou des modes, dans une recherche qui court chez lui de ses proses de jeunesse à ses pièces de théâtre impossibles.

Une voie de la prose qui a certainement partie liée avec sa vision de la poésie, exposée dès le seuil des proses d'Impresiones y Paisajes, son premier livre publié en 1918 :

La poesía existe en todas las cosas, en lo feo, en lo hermoso, en lo repugnante : lo difícil es saberla descubrir, despertar los lagos profundos del alma. Lo admirable de un espíritu está en recibir una emoción e interpretarla de muchas maneras, todas distintas y contrarias ${ }^{2}$.

2 Puisque la poésie existe en toute chose et que l'esprit du poète reçoit et interprète le pluriel, la prose labile et multiforme peut accueillir ce qui est, mais qu'il est si difficile de saisir. La prose, « libre et dure $»^{3}$, devient le lieu du saisissement de cette poésie qui passe parmi nous :

La poesía es algo que anda por las calles. Que se mueve, que pasa a nuestro lado. Todas las cosas tienen su misterio, y la poesía es el misterio que tienen todas las 
cosas. Se pasa junto a un hombre, se mira a una mujer, se adivina la marcha oblicua

de un perro, y en cada uno de estos objetos humanos está la poesía ${ }^{4}$. organisées autour de six «grands" poèmes en prose identifiés comme tels par la critique, qui suit en cela les annotations de l'auteur. Ces proses sont également peu envisagées comme un ensemble cohérent. La raison première en est l'état parfois fragmentaire et inachevé de certains textes qui nous sont parvenus grâce aux efforts des chercheurs. D’autres raisons sont les considérations hiérarchiques conscientes ou non entre poésie en vers et poésie en prose, entre recueils et textes isolés, entre œuvres de jeunesse et de maturité. Les proses poétiques des années 20 ont d'ailleurs longtemps souffert d'un déficit d'attention par rapport à la poésie en vers de la même époque, phénomène qui commence à être rectifié aujourd'hui ${ }^{8}$. Parfois, certains textes redécouverts ou étudiés souffrent de ce dont ils manquaient au départ, quand la caractérisation, sous couvert d'intégrer les textes à un courant, masque la continuité d'un processus d'écriture et de recherche via la prose, comme cela a pu être le cas de la singularité de la voix lorquienne.

6 Cependant, on doit à Andrew A. Anderson et Encarna Alonso Valero, respectivement en 2000 et $2007^{9}$, l'heureuse édition de certaines proses de Lorca. Leur approche générique varie: alors que Anderson publie ce qu'il nomme les «Poemas en prosa » - où il rassemble six textes achevés et publiés par Lorca, tous décrits entre mi-1927 et fin 1928, et cinq textes incomplets, de la même époque -, Alonso Valero fait le choix d'un tour surréaliste - Pez, astro y gafas -, appliqué alors à un ensemble de textes réunis sous le nom de " prosa narrativa breve ». Les deux éditions présentent, dans le même ordre, les textes suivants : "Santa Lucía y San Lázaro ", " Nadadora sumergida ", "Suicidio en Alejandría », "Amantes asesinados por una perdiz ». Elles divergent ensuite puisque «Degollación del Bautista» apparaît avant «Degollación de los Inocentes » chez 
Anderson, et se distinguent totalement pour ce qui est du reste de l'ouvrage : Anderson réunit sous le titre d'« Apéndice » les textes «Cœur azul. Corazón bleu », "Corazón bleu y cœur azul ", « Meditaciones a la muerte de la madre de Charlot », "La gallina », " Mi amor en el baño »; Alonso Valero publie quant à elle «La gallina ", « Historia de este gallo », «En el jardín de las toronjas de luna. Prólogo », « Meditaciones y alegorías del agua ", " Poemas heroicos ", "Las serpientes ", "Telégrafo ", "Árbol de sorpresas ", " Juego de damas », "Santa Liria », « [Un leñador con muchos hijos] », « La muerte de la madre de Charlot ", " "Cœur" azul - corazón "bleu" ». Soient 11 textes pour le premier et 19 pour la seconde, qui inclut des textes rédigés par Lorca entre 1920 et 1922. Les Obras completas, préparées par Miguel García Posada, nous offrent cependant d'autres proses poétiques et poèmes en prose, dont « Paseo », «Barcarola », « Los grillos », « [La noche centenaria] » et «[Lanzando piedras al viento] ». Ce dernier avait d'ailleurs été publié en traduction, dans l'ouvrage édité par André Belamich en 1981 dans La Pléiade, qui a longtemps servi de référence au vu des aléas dont furent victimes les manuscrits lorquiens ${ }^{10}$.

7 D'un point de vue biographique, ces proses sont précédées par des textes composés en 1917 et 1918. Ainsi sans ses Místicas (de la carne y el espíritu), textes annoncés comme « en préparation" dans la liste d'œuvres d'Impresiones y paisajes, le jeune Federico expérimente l'expression des liens et des tensions entre mysticisme et désir charnel au biais d'une prose qu'il forge comme un espace de visions oniriques entrecoupées de réflexions transcendantales religieuses et de prières. Un mysticisme qui n'a rien de la quiétude puisque fait de conflits enchâssés, de critiques et de révoltes contre la société, l'Église, le mystère du corps. Ces premières proses composées entre mai et octobre $1917^{11}$ présentent des éléments que l'on retrouvera dans toutes ses proses poétiques à des degrés divers, tels que la "vision », la «méditation » ou le dialogue, central pour certaines, comme c'est le cas du «Diálogo espiritual » entre Satan et « un alma » dans "Mística doliente» ou de celui entre Platon et Sappho qui s'associe à la vision présentée dans «Poema de la carne. Nostalgia olorosa y ensoñadora", au sous-titre biffé : «Estado sentimental ». Dans ses « états sentimentaux », il explore le terrain de l'écriture subjective, tour à tour et tout à la fois romantique, impressionniste et symboliste. Au travers du motif du rêve, qu'il emprunte à Novalis pour ses choix thématiques et stylistiques, et dans des échos nervaliens, Lorca s'inscrit dans une pratique créative qui affirme que la prose est synonyme de poésie ${ }^{12}$. Ces compositions datées de l'automne 1917 et de janvier 1918 sont remarquables également pour l'hésitation manifeste entre des titres multiples ou raturés, qui montrent la recherche en cours et renforcent la difficulté qu'il y a à les étiqueter: "Canción ", "Visión ", «Sinfonía mágica », « Ensueño », « Sueño despierto », « Sonata » ${ }^{13}$.

$8 \mathrm{Au}$ cœur du processus superposé de création qui est celui de Lorca - il travaille sur plusieurs textes, formes, genres, projets à la fois -, l'écriture en prose poétique se poursuit donc dans les années 20 , comme l'ont montré les divers chercheurs qui ont sauvé de la disparition la plupart des proses que nous pouvons aujourd'hui consulter ${ }^{14}$. Et culminerait pour certains, dans une vision parfois téléologique de l'histoire littéraire sinon de la créativité, dans les poèmes en prose avant-gardistes voire surréalistes ${ }^{15}$ des années 1927 et 1928. S'ils ne sont pas publiés en ouvrage avant 2000, et jamais de son vivant, ces textes furent diffusés précédemment dans des revues : «Santa Lucía y San Lázaro ", en décembre 1927 dans la Revista de Occidente ; "Nadadora sumergida » et "Suicidio en Alejandría », avec des dessins de Lorca, en septembre 1928 dans L’Amic de les Arts ; «Degollación de los Inocentes », le 15 janvier 1929 dans La Gaceta literaria avec 
une illustration de Dalí ; «Amantes asesinados » avec le sur-titre «Autoretrato » dans la revue Ddooss de Valladolid en mars 1931 ; «Degollación del Bautista » dans la revue Avance de La Havane en avril 1930 ; à ces six poèmes en prose l'on peut ajouter « La gallina », publié en mai 1934 dans La Revista Quincenal de Vitoria.

9 En 1927, en pleine effervescence surréaliste, Lorca fait le choix du retour à la prose, d'un réinvestissement de la poésie en prose. Cette année est pour lui l'« année Dalí » : il lui rend visite à Figueras en Catalogne, ce dernier l'initie au cubisme alors que ses expositions catalanes sont un succès et prépare les décors de Mariana Pineda, dont la première a lieu en juin à Barcelone. C'est l'année de la rencontre de Lorca avec Lluis Montanya - à qui il dédie « La degollación del Bautista » - et avec Sebastià Gasch - à qui il dédie "Santa Lucía y San Lázaro » - ; de sa collaboration avec la revue avant-gardiste de Sitges L'Amic de les Arts, qui publie en juillet le «Saint Sébastien » que Dalí rédige en prose et dédie à Lorca ${ }^{16}$. Ce dernier témoigne alors de l'enthousiasme que la lecture de cette prose a suscité en lui : "Se trata sencillamente de una prosa nueva de relaciones insospechables y sutilísimos puntos de vista ${ }^{17}$ ». 1928, quant à elle, est l'année où la créativité de Lorca suit à nouveau des sentiers multiples. En octobre est jouée Mariana Pineda à Madrid, en novembre est publié le poème en prose "Santa Lucía y San Lázaro »; Lorca cherche à éditer ses dessins; il travaille sur La Zapatera prodigiosa, sa farce aux tours symbolistes, mais compose aussi les poèmes «Oda al Santísimo Sacramento del Altar ", et " Oda a Sesostris ». Lorca lance le premier numéro de gallo à Grenade en mars, qui a pour objectif d'être une revue d'avant-garde sœur de la revue catalane, pour laquelle il traduit avec d'autres le «San Sebastiá » de Dalí en castillan et compose «Historia de este gallo». En juillet 1928, c'est la publication et le succès immédiat du Romancero Gitano, mais Lorca affirme se trouver déjà bien loin de ces textes: à l'automne il travaille sur «Nadadora sumergida» et "Suicidio en Alejandría ». Il donne à la même époque sa conférence "Imaginación, inspiración et evasión » à Grenade, publiée dans El defensor de Granada le 11 octobre 1928. Puis, le 15 janvier 1929, il publie «La degollación de los Inocentes » en première page de La Gaceta Literaria avec un dessin de Dalí et un poème en prose de Buñuel. C'est également cette année qu'il écrit "Amantes asesinados por una perdiz », conçu comme un hommage à Guy de Maupassant et aujourd'hui inséré dans le recueil posthume Poeta en Nueva York ${ }^{18}$.

Mon propos n'est pas ici de retracer l'histoire de ces textes, qui a été remarquablement établie, ni de les passer au crible d'une analyse visant à les insérer dans tel ou tel courant artistique. L'élaboration de critères est toujours problématique à l'heure de définir l'« appartenance » des proses des années 20 dans des catégories plus fines que celle englobante des avant-gardes, comme peuvent en faire l'expérience les éditeurs d'anthologies: ainsi, alors que Ródenas sélectionne seulement les deux «Degollaciones» pour les faire figurer dans Prosas del 27, Millares place quant à elle " Nadadora sumergida ", « Suicidio en Alejandría ", «Degollación de los inocentes » et "Asesinados por una perdiz» dans la section «Prosas expérimentales, lúdicas y visionarias », alors qu'existe dans son anthologie la rubrique « Microrrelatos, cinegrafia y poemas en prosa ». Les catégorisations sont certes indispensables à la clarté, mais elles peinent à rendre compte de l'exercice de liberté que constitue pour Lorca l'écriture en prose, polymorphe et déliée. Elles sont d'autant plus difficiles à établir que le corpus établi ne correspond pas à une vision linéaire de la création, qui procèderait par étapes créatives marquées et différenciées, mais plutôt à une vision palimpseste de la créativité et des formes d'expression. La disparité est alors de mise au sein du corpus, surtout si l'on songe à la versatilité des textes concernés, à leur état fragmentaire 
parfois, à leur brièveté et, surtout, à leur singularité anticonformiste. Ces textes, longtemps considérés par la critique comme des hapax dans l'œuvre lorquien, ou comme une marche vers un aboutissement qui serait celui de Poeta en Nueva York et vers une étape dramaturgique conçue comme résolution des conflits intimes et sociaux dans la sublimation que serait une écriture de l'impossible représentativité, ne sont en rien des "brouillons", " études » ou " essais ». Ils ne peuvent être réduits à ce potentiel négatif ou identifiés comme à des éléments dispensables à l'aune de la critique de l'œuvre lorquien : ils sont porteurs au contraire d'un projet poétique réel et constitutif d'une pratique constamment continuée et reprise de la création.

11 Lorca fait en 1927 le choix d'un déport vers la prose qui n'est en rien une nouveauté pour lui. Il est conscient de l'attractivité des «prosas » dans les revues ${ }^{19}$ et déploie en début d'année son activité en ce sens, comme le prouve sa lettre à Guillermo del Torre de février :

[...] estoy copiando varios diálogos en prosa que tengo, que irán bien en la Gaceta. Diálogo de Búster Keaton, diálogo fotografiado, etc. Prefiero publicar prosas. A la Revista de Occidente voy a enviar unos ensayos en prosa y en la Gaceta quisiera debutar así. ¿ Te parece bien ?... ¿ o deseas al vate ?20.

12 La dissociation entre "vate » et prosateur est ironique car il ajoute immédiatement: "son distintas muestras de mi lira». En opérant ce retour vers la prose poétique, Lorca affirme composer des "poemas »: "En prosa hago ahora un ensayo en el que estoy interesadísimo. Me propongo dos temas literarios, los desarrollo y luego los analizo. Y el resultado es un poema ${ }^{21} »$. S'il décrit modestement ses recherches en prose, et parle de «Santa Lucía y San Lázaro comme d'« un ensayo en prosa »22, Lorca se trouve dans une forme d'expérimentation qu'il formule progressivement avec plus de clarté. Il écrit en juillet: « He trabajado bastante en nuevos y originales poemas pertenecientes ya, una vez terminado el Romancero gitano, a otra clase de $\cos ^{2} s^{23}$ ", avant de finir par affirmer à Gasch en septembre, sur un ton polémique : "Y me da horror el poema con versos " ${ }^{24}$. La réception du Romancero Gitano joue un rôle dans cette reprise de la prose. Lorca veut en effet s'éloigner de l'image que l'on donne de lui dans la lecture du Romancero Gitano, et écrit la plupart de ses poèmes en prose lorsqu'il est « victime » du succès du recueil. Il s'en plaint et explique sa situation dans une lettre à Guillén de janvier 1927 :

Me va molestando un poco mi mito de gitanería. Confunden mi vida y mi carácter. No quiero, de ninguna manera. Los gitanos son un tema. Y nada más. Yo podía ser lo mismo poeta de agujas de coser o de paisajes hidráulicos. Además el gitanismo me da un tono de incultura, de falta de educación y de poeta salvaje que tú sabes bien no soy. No quiero que me encasillen. Siento que me van echando cadenas. NO (como diría Ors ${ }^{25}$.

Le refus des définitions et des étiquettes est catégorique. Il prend la forme d'un emprunt-pastiche des Gloses d'Eugeni d'Ors et sa virulence touche à deux éléments : Lorca n'est en rien un poète " naturel », " spontané », sauvage dit-il, mais au contraire un adepte du travail, de l'effort et de la discipline dont sont témoins ses correspondants à qui il raconte combien il travaille ${ }^{26}$. Une revendication que l'on retrouve également dans la formulation de sa poétique transmise par Gerardo Diego : «si es verdad que soy poeta por la gracia de Dios - o del demonio - también lo es que lo soy por la gracia de la técnica y del esfuerzo, y de darme cuenta en absoluto de lo que es un poema ${ }^{27} »$. Lorca est de plus un écrivain cultivé, dont l'écriture est pétrie de lectures, réflexions et références précises subtilement agrégées à sa créativitén ${ }^{28}$. Le deuxième point qu'il entend marquer est qu'il y $\mathrm{a}$ un monde entre le thème de ses poèmes et sa poétique. Les 
gitans peuvent aisément être remplacés par des aiguilles ou des mécanismes hydrauliques: c'est d'ailleurs ce qu'il se propose de faire dans ses poèmes en prose. Lorca entend pour cela montrer la diversité de ses créations pour contrer l'image de poète gitan qui occulte son projet poétique :

Mis libros ya van a salir. Para muchos serán una sorpresa. Ha circulado demasiado mi tópico de gitanismo, y este libro de Canciones, por ejemplo, es un esfuerzo lírico sereno, agudo, y me parece de gran poesía (en el sentido de nobleza y calidad, no de valor). No es un libro gitanístico ${ }^{29}$.

14 En 1928, Lorca est à même de parler plus précisément de ses nouvelles créations. Ainsi confie-t-il à Jorge Zalamea que sa poésie est désormais une poésie "de abrirse las venas ", une poésie violente et agonique qui mêle amour et ironie :

He trabajado mucho y estoy trabajando. Después de construir mis Odas, en las que tengo tanta ilusión, cierro este ciclo de poesía para hacer otra cosa. Ahora tengo una poesía de abrirse las venas, una poesía evadida ya de la realidad con una emoción donde se refleja todo mi amor por las cosas y mi guasa por las cosas. Amor de morir y burla de morir. Amor. Mi corazón. Así es ${ }^{30}$.

En septembre 1928, il affirme à Gasch "Yo trabajo con gran amor en varias cosas de géneros muy distintos. Hago poemas de todas clases » et « Mi poesía tiende ahora otro vuelo más agudo todavía. Me parece que un vuelo personal $»^{31}$. Puis, dans une autre lettre, quelques jours plus tard et à propos de « Nadadora sumergida » et de "Suicidio en Alejandría » :

Ahí te mando los dos poemas. Yo quisiera que fueran de tu agrado. Responden a mi nueva manera espiritualista, emoción pura descarnada, desligada del control lógico, pero, jojo, jojo! Con una tremenda lógica poética. No es surrealismo, jojo!, la conciencia más clara los ilumina.

Son los primeros que he hecho. Naturalmente, están en prosa porque el verso es una ligadura que no resisten. Pero en ellos sí notarás, desde luego, la ternura de mi actual corazón ${ }^{32}$.

16 La critique s'accorde à dire que cette veine nouvelle a pour déclencheur le "Sant Sebastià̀ ${ }^{33}$ " dalinien et à voir dans les six poèmes en prose " officiels » de Lorca une posture surréaliste. Le dialogisme est indéniable entre Lorca et le Catalan, mais il n'est en rien une adoption inconditionnelle des idées de Dalí ni des principes surréalistes. De plus, les poèmes en prose dialoguent également avec les proses de jeunesse de l'auteur, et les dialogues qu'il a continué à composer. Le résultat en est ce pas de côté qui éloigne Lorca de la poésie versifiée. Mais il ne choisit pas pour autant la voix du surréalisme. Lorca crée des proses qui font trembler la "vieille poésie " mais n'assurent pas pour autant les assises d'une poésie surréaliste. Au contraire, Lorca le détourne et réinvente pour s'en démarquer. S'il en adopte les procédés de décomposition et de fragmentation du réel, ce n'est pas sous le coup d'une révélation qu'il lui serait extérieure. Ses proses les plus anciennes explorent déjà la diffraction et la perspective, comme c'est le cas dans « Meditaciones y alegorías del agua » :

Hace muchos años yo, soñador modesto y muchacho alegre, paso todos los veranos en la fresca orilla de un río. Por las tardes, cuando los admirables abejarucos cantan presintiendo el viento y la cigarra frota con rabia sus dos laminillas de oro, me siento junto a la viva hondura del remanso y echo a volar mis propios ojos que se posan asustados sobre el agua, o en las redondas copas de los Álamos.

Bajo las mimbres picadas, y junto a la lengua del agua, yo siento cómo toda la tarde abierta hunde mansamente con su peso la verde lámina del remanso [y] cómo las ráfagas de silencio ponen frío el asombrado cristal de mis ojos.

Los primeros días me turbó el espléndido espectáculo de los reflejos, las alamedas 
caídas que se ponen salomónicas al menor suspiro del agua, los zarzales y los juncos que se rizan como una tela de monja.

Pero yo no observé que mi alma se iba convirtiendo en prisma, que mi alma se llenaba de inmensas perspectivas y de fantasmas temblorosos ${ }^{34}$. leur capacité d'aventure est intacte lorsque l'on cesse de s'intéresser au lien logique entre les choses et que l'on brise les amarres du visible et de l'invisible ${ }^{35}$, Lorca affirme par ailleurs que ses créations répondent à une « terrible logique poétique » et que « la conscience la plus claire les illumine ». Il est donc exemplaire de la réception singulière du surréalisme en Espagne, mouvement qui stimule sa créativité mais ne l'influence pas complètement.

C'est dans la conjonction entre rénovation esthétique, rendue possible par les avantgardes et le surréalisme, et choix de la prose que se situe l'acte subversif chez Lorca. Comme l'affirme Alonso Valero, ses poèmes en prose figurent parmi «sus obras de mayor transgresión $»^{36}$, ce sont des subversions poétiques qui parlent d'une poésie à détruire, d'une poésie en voie de disparition, et cela à travers une forme, le poème en prose, qui après 1929 disparaîtra de l'œuvre lorquien. Mais n'oublions pas que la subversion était déjà présente et puissante dans les Místicas et les Estados Sentimentales, dont la portée critique s'unissait à la rêverie de soi au sein de paysages oniriques fantaisistes, de voyages extraordinaires et de dialogues faisant résonner les voix des marginaux et déchus. Or, la prose poétique et le poème en prose incarnent la dissonance, le geste y est moderne mais également conscient du passé, de ses traces et de sa disparition. Ces textes, neutralisés quand ils sont réduits à des essais surréalistes ou de jeunesse, sont le lieu d'une prose tremblée, d'une prose comme cours et creuset de l'expérimentation, de l'hybridation, de la rupture. En eux Lorca construit une poétique du tremblement et un dire autre qui crée (sur) la disparition et le conduira à la saisie du vide et du creux dans Poeta en Nueva York ${ }^{37}$.

\section{Une créativité de la disparition}

19 Les images de la disparition sont constantes dans ce corpus. Elles composent une atmosphère faite de départs, d'au revoir, de dissolutions et d'éclipses, et se déploient sur une gamme étendue qui part de l'intermittence pour rejoindre la disparition. En cela les textes donnent corps à l'ensemble des acceptions du terme "disparition », qui désigne ce qui cesse momentanément d'être visible, mais aussi ce qui s'éloigne, s'absente, s'égare ou est égaré, se perd ou est volé, et ce qui cesse d'exister. Or, les notions évoquées dans ses définitions - invisibilité, éloignement, absence, égarement, vol et destruction -, ont toute partie liée avec une écriture qui est une recherche, un acte de traduction d'un réel en cours de disparition.

Se multiplient alors clignements et étincellements, comme par exemple dans le très beau " Telégrafo » où le sujet poétique qui rêve à un pays absent et lointain est surpris par l'union des clignements des étoiles et du télégraphe :

Miré al cielo indolentemente y vi que todas las estrellas telegrafiaban en el infinito con sus parpadeos luminosos. Sirio sobre todas ellas enviaba tics anaranjados y tacs verdes entre el asombro de todas las demás.

El telégrafo luminoso del cielo se unió al telégrafo pobre de la estación y mi alma (demasiado tierna) contestó con sus párpados a todas las preguntas y requiebros de las estrellas que entonces comprendí perfectamente ${ }^{38}$. 
21 lointain, mais dont l'éclat demeure dans la pupille et dans l'être. Cet instant rare est celui de la captation du mystère, qui s'offre subrepticement à l'œil : si Psyché dans les « Poemas heroicos ", qui se baigne dans la pupille tremblante d'un bois discret, à l'heur de voir "cómo las estrellas abren sus párpados blancos", elle pleure pourtant la disparition d'un autre clignement de paupières, celles des ailes du papillon venu boire sur ses lèvres de vierge : « ¡Oh, Mariposa ! - dice -, vete invisible y vuelve invisible [...] y yo seré como un arpa yacente que, pulsada por la brisa, llore tu ausencia $»^{39}$. Ce même papillon dont la métamorphose vole aux dames l'objet de leur désir dans «Juego de damas » :

Las cinco damas de una corte llena de color y poesía, enamoradas las cinco de un joven misterioso que ha llegado a ella de lejanas tierras. Lo rondan, lo cercan y se ocultan mutuamente su amor. Pero el joven no les hace caso. El joven pasea el jardín enamorando a la hija del jardinero ${ }^{40}$, joven con la piel tostada y de ninguna belleza, aunque sin fealdad, desde luego. Las otras damas lo rondan y averiguan de qué se trata e, indignadas, tratan de matar a la joven tostada, pero cuando llegan ya está ella muerta con la cara sonriente y llena de luz y aroma exquisito. Sobre un bando del jardín encuentran una mariposa que sale volando y las ropas del joven.

La jeune fille au visage dérobé par la brûlure recouvre la beauté de ses traits dans la disparition ultime, alors que du jeune amoureux désiré par les dames ne persiste que l'habit : les deux corps disparaissent dans deux métamorphoses qui les séparent l'un de l'autre après la fulgurance de la rencontre amoureuse.

Les paysages tremblent dans les premières proses poétiques des années 20 , ils frémissent, rendus intermittents par le chant des cigales et le palpitement de la lumière : « Por el aire yacente de la noche estival flotaban las temblorosas cintas de los grillos». Devenus espaces de changements, ils accueillent la disparition en cours: « miré a la copa real que estaba inundada de sol poniente y sólo los invisibles pajarillos del viento jugaban entre las hojas; el pájaro de oro había desaparecido $»^{41}$. L'émotion peut également surgir des êtres et des choses qui ont disparu sans même avoir été :

Una emoción aguda y elegíaca por las cosas que no han sido, buenas y malas, grandes y pequeñas, invade los paisajes de mis ojos casi ocultos por unas gafas de luz violeta. Una emoción amarga que me hace caminar hacia este jardín que se estremece en las altísimas llanuras del aire ${ }^{42}$.

Dans ce texte apparaitt le motif récurrent du voyage. Transformation et disparition sont alors deux faces d'un même événement, lorsque le voyageur traverse des espaces en cours de métamorphose, les laisse disparaître derrière lui et s'engage sur une voie qui n'est jamais évidente, claire et précise, mais au contraire floue, déjà effacée dans l'instant où il avance. Le voyageur des proses lorquiennes est souvent sur ce « camino que va a ninguna parte, donde están los que han muerto esperando $»^{43}$. En tous les cas, il fait partie de ces êtres en partance qui peuplent les proses, à l'image des bancs de «Paseo » qui partent « en busca de otros paisajes y otros ritmos » ${ }^{44}$. Il se prépare pour un déplacement, quitte lieux et choses, dans des textes qui s'ouvrent sur des au revoir : "Me he despedido de los amigos que más quiero para emprender un corto pero dramático viaje. Sobre un espejo de plata encuentro, mucho antes de que amanezca, el maletín con la ropa que debo usar en la extraña tierra a que me dirijo ». Ces mots, qui constituent le début de qui était prévu comme le prologue de «En el jardín de las toronjas de luna », reviennent transformés dans les dernières lignes de «Santa Lucía y San Lázaro » : 
Hace unos momentos que estoy en casa.

Sin sorpresa he hallado mi maletín vacío. Sólo unas gafas y un blanquísimo guardapolvo. Dos temas de viaje. Puros y aislados. Las gafas, sobre la mesa, llevaban al máximo su dibujo concreto y su fijeza extraplana. El guardapolvo se desmayaba en la silla en su siempre ultima actitud, con una lejanía poco humana ya, lejanía bajo cero de pez ahogado. Las gafas iban hacia un teorema geométrico de demostración exacta, y el guardapolvo se arrojaba a un mar lleno de naufragios y verdes resplandores súbitos. Gafas y guardapolvo. En la mesa y en la silla. Santa Lucía y San Lázaro ${ }^{45}$.

Ce qui était l'aune d'un départ dans le premier texte est ici devenu un adieu, adressé au voyage lui-même puisque le voyageur est revenu chez lui, a pris le train qui l'éloigne de l'étrange ville marine où les magasins d'optique réfractent les yeux de Sainte Lucie et où, dans la gare, il a croisé la figure hiératique et suspendue de San Lázaro. Adressé également aux lunettes et à l'imperméable, présents sur les meubles de la chambre mais déjà absents et lointains. Or, ces objets, métaphores et signes de ce que Lorca laisse disparaitre derrière lui, sont au cœur de sa représentation critique et de sa volonté de redéfinition esthétique ${ }^{46}$.

La critique s'est intéressée à ce que, à la suite d'Antonio Monegal, on a nommé "rhétorique de la violence, de la mutilation et du sang ${ }^{47}$ dans les poèmes en prose de Lorca. L'analyse de la "cruauté » dans les textes se fait alors en termes métapoétiques et manifeste les attaques virulentes de Lorca contre le monde figé de la poésie pure et contre la sociétét8. Ces éléments sont indéniables, mais je m'intéresserai ici davantage à la persistance de la disparition dans les textes, à l'écriture du processus même de la disparition, et donc à ses traces. Elles actualisent le processus de recherche créative de Lorca à chaque lecture : en effet, ces marques ne disparaitront jamais totalement car elles sont aujourd'hui déposées, transposées dans les poèmes. Aussi paradoxal que cela puisse être, il leur faut un témoin, un lecteur qui fasse que ces indices de la disparition existent, qu'ils deviennent la marque d'une permanence que le texte rend possible. La disparition, malgré les sens que le dictionnaire lui accole, n'est donc jamais élimination ${ }^{49}$.

Elle n'est pas non plus strictement associée aux éléments du passé jugés inutiles et contraignants et ne propose pas une stricte opposition binaire entre l'ancien et le nouveau. Ainsi dans "Santa Lucía y San Lázaro ", c'est avec la même tristesse que le voyageur laisse derrière lui un vieux numéro de Blanco y Negro de 1910 dont la facture et l'idéologie dix-neuviémistes ont été incapables de porter les rénovations esthétiques de cette époque, qui sont déjà vieilles, et les noms accolés des papiers à cigarettes, dans un jeu qui est une composition surréaliste déjà distante et abandonnée par Lorca: "Tristeza recién llegada de los librillos de papel marca "El Paraguas", "El Automóbil" y "La Bicicleta"; tristeza del Blanco y Negro de $1910 »^{50}$. À cette association des signifiants, Lorca préfère le lien poétique qu'il établit entre les lunettes et l'imperméable : en cela ce poème en prose est, depuis le surréalisme, et déjà, une critique du surréalisme en littérature.

Dans cette poésie "à s'ouvrir les veines ", ce sont les corps qui disparaissent. Ou plutôt leur unité organique, comme dans « Mi amor en el baño » :

Al entrar en el cuarto de baño vi a mi amor que se dividía para no quererme.

El sistema de venas latía sobre un maniquí. El sistema muscular pendía de los tubos hirientes. El sistema muscular pendía de los tubos hirientes. El sistema nervioso saltaba sobre une rana. El sistema de aire lloraba. Cuatro sistemas y su sombra en la bañera pura ${ }^{51}$. 
poésie est celle de la destruction en cours, de la disparition créee. Les morts ne partent jamais vraiment, ne quittent pas les textes, qu'ils habitent tel l'enfant au visage blanc de Poeta en Nueva York. Les corps morts non plus, et leur incarnation maximale est celle des corps saints. La présence des saints et l'adoration de corps putréfiés ${ }^{52}$ est un phénomène récurrent déjà dans son œuvre antérieure à 1927. Avant même d'être lié à ce que les siècles et l'Église ont consacré par le pouvoir de l'image, le saint est un corps $^{53}$. Un corps en souffrance, mais une souffrance sans douleur, car lors de son martyre il est habité par l'esprit Saint et ne ressent aucune des tortures qu'on lui afflige. Il est aussi un corps démembré, découpé, éparpillé lorsque les restes deviennent reliques et que sont fondés les loci, les lieux d'adoration du saint qui vont progressivement structurer la géographie de la chrétienté. Le saint, c'est le corps d'un être humain, d'un semblable, qui par ses actes et sa mort devient médiateur entre l'humain et le divin. Étudier le culte des saints c'est comprendre le rôle que des humains morts jouent dans le contact entre le ciel et la terre. Tout comme l'écriture est ce lien aux disparus et aux ombres du passé, contact également avec une tradition, une culture, des formes d'expression à ingérer et dépasser dans le même geste créateur. Car le saint, c'est aussi un texte : la "vie » du saint, reprise le jour où il est célébré, forme de légende acceptée par le dogme, qui actualise à chaque fois le martyr et le miracle, qui crée du symbolique et du lien. Les recueils de vies des saints, songeons à La Légende dorée de Jacques de Voragine (1265) que Lorca connaît parfaitement et réécrit, sont la manifestation par laquelle la culture savante écrite accepte le plaisir du même et de la variation né tout d'abord dans la culture orale. Ils constituent un trésor d'invention que l'artiste réinvestit.

Si l'on cherche les traces des saints chez Lorca, on voit se dessiner une écriture de l'être-disparaître, de l'être en disparition. Un poème de jeunesse s'attache au saint patron de l'Espagne, Saint Jacques, le jour de sa fête, le 25 juillet 1918 : Santiago n'est cependant pas créé dans sa version guerrière, ce n'est pas un poème de circonstance. Il est le voyageur qui passe et part, il est une disparition à laquelle est liée le désir de dissolution du poète.

Esta noche ha pasado Santiago

su camino de luz en el cielo.

Lo comentan los niños jugando

con el agua de un cauce sereno.

¿Dónde va el peregrino celeste

por el claro infinito sendero?

Va a la aurora que brilla en el fondo

en caballo blanco como el hielo.

**

¡Noche clara, finales de julio!

¡Ha pasado Santiago en el cielo !

La seule trace du passage du saint est la tristesse de l'âme laissée sur le chemin devenu blanc, de la couleur de son cheval, comme offrande aux jeux et au vent qui pourront la disperser. Les poèmes sur les saints prennent ensuite forme dans une écriture des lieux : comme si le loci du saint s'incarnait dans les topoi du poème. Ainsi le Romancero Gitano propose trois poèmes sur trois villes, les trois joyaux de l'Andalousie, Grenade, Cordoue et Séville, et trois saints qui, plus que des saints, sont des archanges : et la différence est d'importance. L'archange est séparé de l'humain, il est de l'ordre du divin. Mais Lorca les tire vers l'humain. Dans «San Miguel, Granada », le justicier, traditionnellement représenté l'épée à la main, est métamorphosé, son corps 
recomposé dans une «burla» ironique qui part de l'image de la statue de Miguel à Grenade, baroque et revêtue de dentelle, et débouche sur le portrait sensuel d'un éphèbe perdu :

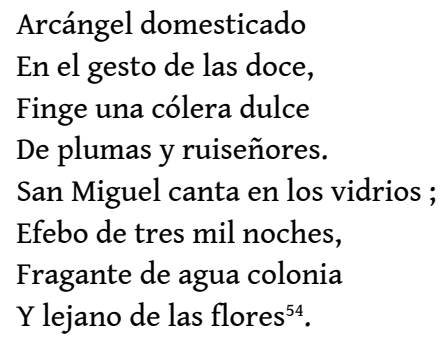

31 La contamination entre la cérémonie religieuse et une scène païenne accompagne la solitude de Miguel, finalement isolé dans sa tour sur le Sacromonte : «San Miguel se estaba quieto / en la alcoba de su torre, / con las enaguas cuajadas / de espejitos y entredoses ». Dans "San Rafael, Córdoba », le lecteur peut longtemps se demander où se trouve le guérisseur. Le poème est consacré au fleuve, Guadalquivir, aux deux Cordoue qui coexistent et aux enfants qui les habitent. Le corps du saint est presque absent :

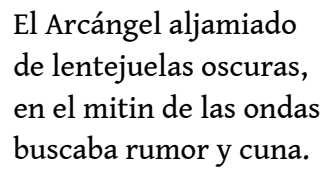

Il se caractérise par sa position au partage des eaux ; il est le poisson qui relie les villes et le représente traditionnellement: "Un solo pez en el agua / que une a las dos Córdobas ». Le glissement fait du corps du saint ce point de contact disparaissant dans les eaux qui séparent et unissent :

Un solo pez en el agua.

Dos Córdobas de hermosura.

Córdoba quebrada en chorros.

Celeste Córdoba enjuta ${ }^{55}$.

Dans "San Gabriel, Sevilla ", le messager se retrouve pris dans un poème qui récrit l'Annonciation à la Vierge. Gabriel rencontre Anunciación, jeune sévillane. Il lui annonce un fils, et la souffrance de cet enfant : « Tu niño tendrá en el pecho / un lunar $\mathrm{y}$ tres heridas ", "Tres balas de almendra verde / tiemblan en su vocecilla ». Ce poème narratif, qui commence sur la vision d'un adolescent gitan, Gabriel, et se clôt sur son départ, offre un dialogue savoureux d'Andalous. Gabriel écrase presque, dans sa modernité, son humanité gitane et sa fusion avec la nature, la jeune femme dont le corps à l'instant fécondé portera l'enfant qui naîtra pour être blessé et mourir. L'impassibilité du divin incarné dans cet ange promeneur, résonne avec l'image obsédante dans l'œuvre de Lorca de la mort qui marche «en souliers vernis ». Ces recréations des corps des saints dans la fusion du légendaire et de l'acte poétique nouveau, annoncent la disparition.

Il manque ici un archange : traditionnellement ces derniers sont quatre, et l'absence d'Uriel signe à la fois le règne verlainien de l'impair et celui du déséquilibre créé par l'absence, le creux. Le déplacement s'opère alors vers la sainte, dans le même recueil : une femme, dans un poème qui oxymorise le discours et rend sensible le contact entre les contraires et les inatteignables. En contrepoint et continuité, "Mártir de santa Olalla » offre aux yeux et à l'oreille la disgrégation violente du corps saint, associé à une autre ville, Mérida : 


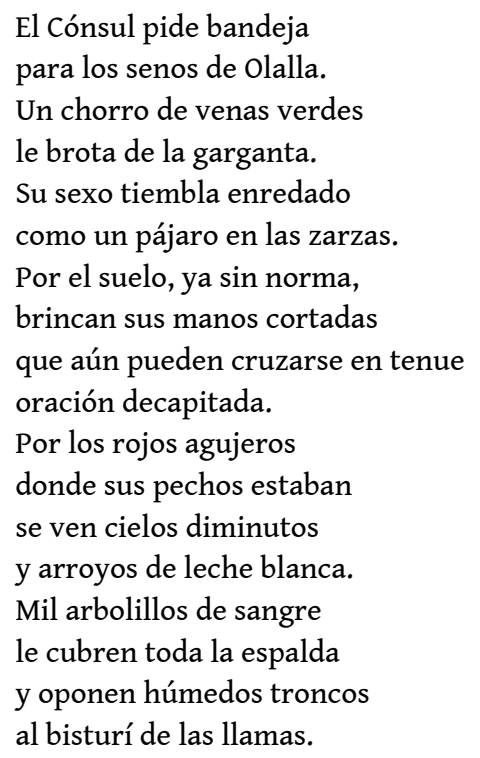

Il s'agit de la recréation du martyr de sainte Eulalie de Mérida, relaté par Prudence au $V^{e}$ siècle dans le plus ancien poème en langue d'Oïl, « la Cantilène de sainte Eulalie ». Lorca respecte toutes les étapes et des détails des tortures infligées et les recrée tout à la fois : frappée par des barres de fer, brûlée par des tisons en ses plaies, seins arrachés jusqu'à l'os, chevelure en flamme. À sa mort, une colombe blanche s'envole des flammes et son corps est recouvert de neige : «Nieve ondulada reposa. / Olalla pende del árbol. / Su desnudo de carbón / tizna los aires helados $»^{56}$. La violence est ici extrême au cœur du poème, la sainte aux seins découpés est brûlée, et le corps noirci devient albe blancheur, dans un renversement final de l'image du corps martyrisé : « Nieve partida comienza. / Olalla blanca en el árbol».

Enfin, et même s'il ne s'agit pas d'un saint chrétien, je ne peux éviter de mentionner le tombeau que Lorca consacre à son ami le torero Ignacio Sánchez Mejías «Cuerpo presente ", dans Llanto por Ignacio Sánchez Mejías. Ce chant funèbre écrit la décomposition du corps ami :

¿Qué dicen? Un silencio con hedores reposa.

Estamos con un cuerpo presente que se esfuma,

con una forma clara que tuvo ruiseñores

y la vemos llenarse de agujeros sin fondo.

L'adieu au corps disparaissant entre ensuite au seuil du recueil en résonnance oxymorique avec le poème suivant, "Alma ausente » qui clôt le chant funèbre et la publication.

L'écriture des traces du corps saint se poursuit et s'intensifie dans les poèmes en prose. Si les saints existent dans les reliques, les loci et les passiones ou récits de leur martyre dans la mémoire chrétienne en tant que "souvenir (installé) dans un tourbillon imaginatif $~_{57}$, Lorca les réactualise et réactive la " puissance agissante des images » qui était celles des icônes et donne une signifiance nouvelle aux codes du visible et de l'invisible qui se tramaient dans le corps saint. Alors qu'il refuse la «terreur domestiquée " par la représentation traditionnelle de la sainteté, il réintroduit une horreur tout humaine dans les images. Il montre en cela qu'il n'y a pas de miracle divin, mais des souffrances réelles. Il brise l'éloignement du divin et du passé. Lorca a cette naturalité à mettre le passé dans le présent, en renversant le sens de la souffrance pour retrouver derrière la passion sans souffrance du saint l'horreur païenne. Il réécrit ainsi 
la douleur des mères rapportées par Augustin en donnant à entendre la décapitation et en déplaçant la scène biblique dans un univers aseptisé de clinique où le bruit de la lame se conjugue à l'odeur du chloroforme :

Tris tras. Zig zag, rig rag, milg malg. La piel era tan tierna que salía íntegra. Niños y nueces recién cuajados. [...] Cuando se vuelvan locas las madres querrán construir una fábrica de sombreros de pórfido, pero no podrán nunca con esta crueldad atenuar la ternura de sus pechos derramados ${ }^{58}$.

Cette nuit de cristal laisse un paysage de sang séché, de mains coupées et de seins inutiles, tandis que s'impose la disparition des vivants. La mort est l'ultime disparition déjouée par la religion catholique dans le culte des saints, qui en éloigne l'insupportable réalité et rappellent constamment le dogme de la résurrection. Or, chez Lorca le saint est en train de disparaître et de mourir, et ses proses questionnent la réalité de la disparition de la douleur et, par ricochet la sainteté telle qu'établie par l'Église. Ainsi les cris de Jean Baptiste sont-ils bien réels, bien que couverts par les clameurs du stade qui ovationne le joueur ayant marqué le but de la victoire: «El griterío del Stadium hizo que las vacas mugieran en todos los establos de Palestina. La cabeza del luchador celeste estaba en medio de la arena $»^{59}$.

La charge critique est puissante dans le réinvestissement d'une signifiance sociale, tout autant que poétique. Ce qui disparaît dans la recomposition du martyr et de son corps dans la prose, c'est aussi le dogme et le sens de la religion catholique. L'inversion est constante dans "Santa Lucía et San Lázaro » : aux yeux de l'Éternel posés sur les Justes (Psaumes 34:15) vont s'opposer les "seis mil dioptrías" qui diffractent les yeux arrachés de Lucie et ne peuvent que refléter un monde de surfaces minérales ; à l'appel à la vie de Jésus se substitue l'attente éperdue d'un Lazare perdu dans une gare : «Le tuve gran lástima, porque sabía que estaba pendiente de una voz, y estar pendiente de una voz es como estar sentado en la guillotina de la Revolución francesa ${ }^{60}$. La décomposition des corps est dans ce texte une recomposition de leurs représentations. Lucía, patronne de la lumière et de la vision, évoquée lors des maladies oculaires, est d'abord écrite au travers du détournement de la tradition iconographique : « La pintan con dos magníficos ojos de buey en una bandeja ». Ces yeux bovins sont les siens, arrachés pendant son martyre avant que la Vierge ne lui en apporte de plus beaux. Pour remercier sainte Agathe d'avoir rendu la santé à sa mère, Lucie de Syracuse a fait vœu de chasteté et de pauvreté et donné tous leurs biens. Mais sa mère l'avait promise à un jeune homme qui réagit mal et la dénonce comme chrétienne au consul Pascasius qui veut la placer dans un lupanar. Son corps est alors transformé en bloc inamovible par le Saint Esprit, même tirée par des bœufs ; il reste ensuite indemne malgré la poix, l'huile brûlante, le bûcher. Lucía meurt enfin, une épée dans la gorge. Lorca récrit son martyre ${ }^{61}$ et fait disparaître les yeux divins de son texte. Seuls demeurent les yeux arrachés, qui n'ont d'autre utilité que de composer des natures mortes qui ne sont guère vivifiées par leur surréalisme puisque toujours nées de l'association d'éléments qui disparaissent ou sont tronqués. Au moment où Lorca s'empare du surréalisme, il le dépasse et le critique dans ses poèmes en prose :

Ojos de Santa Lucía sobre las nubes, en primer término, con un aire del que se acaban de marchar los pájaros.

Ojos de Santa Lucía en el mar, en la esfera del reloj, a los lados del yunque, en el gran tronco recién cortado.

41 Au cœur de la métamorphose lorquienne, les images héritées disparaissent donc, dans un retour sur les représentations transmises. Le jeu est identique avec Lazare. De ce 
saint controversé sont célèbres les représentations morbides, dont celle du Caravage, qui a fait une série consacrée à Baptiste, Lazare et Lucie entre 1608 et 1609. Celui qui après quatre jours de décomposition ressuscite à l'appel de son maître est donc le lieu de la représentation de la revitalisation des chairs et de l'esprit. Lorca l'investit et propose un voyage au cœur des représentations. Il reconnaît et dépasse ainsi à la fois la tradition iconographique chrétienne et la révolution picturale opérée au XIX ${ }^{\mathrm{e}}$ siècle : en situant son Lazare dans la "Estación de San Lázaro ", il associe les corps du saint et de la gare. Si la vision est fondamentale dans la disgrégation des éléments traditionnels, l'odorat est le premier sens frappé : la puanteur de Lazare a toujours été un élément délicat à intégrer dans l'iconographie, qui peine à représenter l'odeur d'un corps en décomposition et ne suit guère la Bible dans laquelle on lit que les témoins dissuadent le Christ d'entrer dans le tombeau. Lorca ressuscite cette odeur et la déplace dans des motifs morts et puants, accaparant ainsi la posture thaumaturgique du Christ en réinventant Lazare: "San Lázaro nació palidísimo. Despedía olor de oveja mojada. Cuando le daban azotes, echaba terroncitos de azúcar por la boca $»^{62}$. L'image traditionnelle disparaît et Lazare devient autre: "Después de resucitar inventó el ataúd, el cirio, las luces de magnesio y las estaciones de ferrocarril ». Lorca récupère ici ironiquement une autre tradition iconographique: alors que le corps du saint ressuscité a disparu de l'art, celui de la gare est devenu dans le deuxième tiers du XIX ${ }^{\mathrm{e}}$ siècle symbole de la révolution impressionniste portée par les douze toiles de Monet représentant la gare parisienne de Saint Lazare. Il avait déjà fait de même, quoique de façon plus concentrée, lorsque son voyageur affirme croire en la véracité du voyage fabuleux par les airs de Saint Raymond lors de son retour en Espagne grâce à l'impression que lui procure la façade miraculeuse de la cathédrale de la ville qu'il visite, autre écho de l'œuvre de Monet et de son œuvre-manifeste, les quarante représentations de la cathédrale de Rouen.

Lazare attend la voix qui le sortira de sa léthargie dans un espace de transit et de vide. Lorca reconnaît et fait disparaître deux traditions de représentation dans un même geste créateur, alors même qu'il est tourné vers d'autres révolutions avec lesquelles il dialogue, dont celle du surréalisme, comme le manifeste la dédicace du poème à Gasch. Or, le corps des saints, finalement, disparaît dans ce poème. L'écriture des parts manquantes et des parts tronquées s'associe à celles des lieux et des objets. Après la dislocation ironique des corps qui est une métamorphose en corps poétique pour une poétique de la transgression, ces derniers sont remplacés, dans une espèce de métamorphose moderne, par deux objets: des lunettes et un imperméable. Ces accessoires du voyage et du regard, qui protègent et améliorent les corps, sont la trace ultime de la disparition dans le texte, la marque de corps en souffrance disparus et l'empreinte d'une recomposition esthétique.

Les proses poétiques ont toutes partie liée avec la disparition et la majorité met en scène une mort violente. Si, en 1927, Lorca puise dans le livre sanglant qu'est l'ancien testament et recompose la décapitation de Jean Baptiste et le Massacre des Innocents, ou dans l'actualité parodiée en exposant le meurtre de la comtesse $\mathrm{X}^{* * *}$, têtes décapitées, veines tranchées, mains coupées palpitent dans l'ensemble du corpus. Double suicide, exécutions, décomposition, combat à mort entre une poule et un coq, flots de sang, mouches fouisseuses, corps mutilés, os qui craquent, amants persécutés et assassinés agitent ces cris en prose qui composent une spirale où le fait poétique est vivant, du fait même de créer nouvellement la disparition. La rhétorique est celle de la 
violence nécessaire contre un système de codes littéraires et de normes sociales. Une coupure et un adieu sont en train d'être opérés dans ces textes qui sont la disparition en cours. Aussi Lorca multiplie-t-il les impératifs, qui sont autant d'appel à la disparition :

Es preciso romperlo todo para que los dogmas se purifiquen y las normas tengan nuevo temblor. Es preciso que el elefante tenga ojos de perdiz y la perdiz pezuñas de unicornio ${ }^{63}$.

Había necesidad de romper para siempre. [...] Rompían los tabiques y agitaban los pañuelos. [...] Era de noche, y se hacía precisa la dentadura y el látigo ${ }^{64}$

Era necesario el crujir de los huesos y el romper las presas de los ríos. [...] Es necesario tener doscientos hijos y entregarlos a la degollación. Solamente de esta manera sería posible la autonomía del lirio silvestre ${ }^{65}$.

\section{Conclusion}

Alors que la prose est a priori caractérisée par la fluidité, le lien, l'argumentation, la succession narrative, soit par tout ce qui est fluide et unifie, tel le sang qui coule des textes, de Lorca composés par Lorca à l'orée des années 20 et au cours de cette décennie, deviennent la manifestation d'une poétique de la fragmentation, de la désarticulation et de la disparition comme "chant de l'éloignement " ${ }^{66}$. Ils accueillent le creux dans leur trame, se font acte d'un nécessaire renouvellement de l'écriture et incarnation de cette reprise. Les motifs de cet être-disparaître sont multiples dans des proses où crient l'abîme et la souffrance des corps détruits. Dans un de ses « collaphorismes ${ }^{67}$, Lorca expose le procédé qui sous-tend sa créativité : « Dentro de la palabra estrella están todos los cielos nocturnos que han sido y que serán ». Lorca s'inscrit dans la méditation sur la forme et le langage poétiques impulsée par Baudelaire et Mallarmé : le signe et le signifiant, matières premières du poète, sont déjà une disparition. Ils sont tout à la fois une trace du passé, telles les étoiles disparues depuis longtemps lorsque nous en percevons le clignement, et un guide vers l'avenir quand nous y lisons nos destinées.

Les poèmes en prose sont la trace d'une recherche qui est celle du poème. Et c'est dans son invisibilité que le poème existe: « Ni pompa de jabón, ni bala de plomo. El verdadero poema debe ser invisible ${ }^{68}$. La perfection est donc dans la disparition, et c'est dans l'un de ses poèmes en prose, "Árbol de sorpresas », que Lorca signe son art poétique de la disparition. L'arbre en question est un arbre disparu qui n'apparaît jamais dans ce texte, reprise de l'ambivalent dialogue de jeunesse de Platon sur l'amitié et l'amour homosexuel ${ }^{69}$. Lorca s'attache à la figure de Socrate, qui rejoint d'abord le cercle de ses disciples pour finir par s'en éloigner et disparaître. Le texte se compose de deux parties, séparées et unies par la vision lointaine du jeune Lysis : «A lo lejos Lisis bailaba sobre una pirámide de viento ». Dans la première, l'effeuillement de l'oignon dans le cœur des disciples, face au sourire du maître, incarne la révélation de la vérité sous le signe d'une disparition :

La cebolla se llenó de estrellas doradas y negras como un cielo de retablo y todo dijeron: “¡Ahí está !”... Luego la legumbre ideal fue aminorando hasta que desapareció en finísimas gasas imperceptibles... y todos se fueron.

Dans la seconde, Socrate s'éloigne et regarde le présent qui l'entoure avant d'être marqué par la trace de sa propre disparition, dans un écho christique :

Sócrates vio una luciérnaga, Sócrates oyó un sapo, Sócrates vio una mariposa enorme hacia el Sur... y una culebrilla roja le iluminó todo el pecho, como un 
hachazo de sangre, como una llaga reflejada. Luego se perdió en la avenida de los vientos ${ }^{70}$. disparition du maître va ensuite de pair avec celle de la parole, remplacée par la vision. Les langues des disciples ont beau s'affûter, rien n'est dit, un cri subsiste, et Socrate reste silencieux, regarde, contemple et sourit. Seule persiste dans l'œil du maître l'image du jeune corps agile et dansant de Lysis et les beautés de la nature. Le danseur silencieux, objet du regard et du désir, annonce dans sa volte sur le vent du soir le départ de Socrate sur l'avenue des vents. La recherche est dans la captation de la disparition, au sein d'une forme, le poème en prose, qui chez Lorca sera également amenée à disparaître pour réapparaître dans la création à venir.

\section{NOTES}

1. Miguel GARCíA POSADA, «Introducción », Obras completas de Federico García Lorca, Madrid, Arkal, t. I, p. 12.

2. Federico GARCía LORCA, Impresiones y Paisajes, [1918], Madrid, Cátedra, 2010, p. 57.

3. Id., Interview de 1933 sur Bodas de Sangre, Miguel García Posada (éd.), Obras Completas VI, Madrid, Akal, 2008, p. 534.

4. Ibid., Interview de 1936, p. 728.

5. Loc. cit., « Por eso yo no concibo la poesía como abstracción, sino como cosa real existente, que ha pasado junto a mí ».

6. Ibid., p. 540 .

7. Il s'agit de « Nadadora sumergida » et "Suicidio en Alejandría », «A S. Gasch, mediados de septiembre $1928 »$, O. C. VII, Op. cit., p. 1035.

8. Grâce notamment aux travaux de Domingo RóDENAS, Proceder a sabiendas. Antología de la narrativa de vanguardia española. 1923-1936, Barcelona, Alba, 1997 ; Prosa del 27. Antología, Madrid, Austral, 2000 ; et de Selena MILLAREs, La revolución secreta. Prosas visionarias de vanguardia (ensayo), Santa Cruz de Tenerife, Idea, 2011 ; Prosas hispánicas de vanguardia, Madrid, Cátedra, 2013.

9. Andrew A. ANDERson (éd.), Poemas en prosa de Federico García Lorca, Granada, La Veleta, 2000 ; F. GARCía LORCA, Pez, astro y gafas. Prosa narrativa breve, Encarna ALONSO VALERo (éd.), Palencia, Menos Cuarto, 2007.

10. Federico GARCía LORCA, Euvres complètes I, éd. d'André Belamich, NRF, Gallimard, la Pléiade, [1981], 1986. Notons que Claude Couffon a traduit une de ces proses: Méditations sur la défunte mère de Charlot, édition bilingue, illustrations de Rafael Alberti, Roubaix, Éditions Brandes, 1990.

11. C'est à la fin de la dernière d'entre elles, "Mística en que se trata de Dios ", qu'apparaît cet acte de naissance poétique si souvent cité : « Noche de 15 de octubre de 1917. Federico, 1 año que salí hacia el bien de la literatura ", Prosa inédita de juventud, éd. de Christopher Maurer, Madrid, Cátedra, 1998, p. 152.

12. Hanna JECHOVA-VOISINE, François MOURET, Jacques VOISINE (éds.), La poésie en prose. Des Lumières aux écrivains romantiques (1760-1820), Paris, Presses de l'Université Paris-Sorbonne, 1993.

13. Mon projet d'appréhension critique des proses poétiques de Lorca du début des années 20 et des poèmes en prose de 1927 et 1928 que je me propose de mener me conduira prochainement à 
proposer une relecture de ces textes qui sont à tort séparés alors qu'ils sont extrêmement révélateurs de l'expérimentation continue en prose de leur auteur.

14. Francisco GARCía LORCA dévoile « Meditaciones y alegoría del agua » et " Poemas Heroicos ", de 1918 et 1920, dans Federico y su mundo, Madrid, Alianza, 1993, p. 169-170. André Belamich date «[Lanzando piedras al aire]» des années 20. Eutimio Martín sauve le magnifique « Árbol de sorpresas ", de la même époque. Miguel García Posada nous offre d'autres textes de 1920-1922: « En el jardín de las toronjas de luna. Prólogo », "Serpientes », « Telégrafo », " Juego de damas », et «Santa Liria » de 1924, tandis que «Cœur azul-corazón bleu » de 1928 apparaît dans les O. C. qu'il dirige (Barcelona, Galaxia Gutenberg, 1998, t. III, p. 752). « Corazón bleu y cœur azul », inédit quant à lui jusqu'à l'édition d'Andrew Anderson pour la collection La Veleta aurait été rédigé entre la fin de l'année 1927 et le début de 1928 en réponse au « Pez perseguido por una uva » que Dalí envoie à Lorca probablement en novembre 1927 (Federico García Lorca, Poemas en prosa, op. cit., p. 47). «Meditaciones a la madre de Charlot», brouillon incomplet daté du 7 septembre 1928, dont manquent les pages 7, 10 et 11, est publié par Christopher Maurer dans El País, le 3 décembre 1989. " Mi amor en el baño ", inédit jusqu'à l'édition d'Anderson, est écrit au verso de "Suicidio en Alejandría ».

15. María TERESA PAO, «Con pies de plomo en el arte: Lorca, Surrealism and the Prose Poems », Any Other Path: Spain, Surrealism and Texts Less Considered, Ann Arbor, Michigan, Universidad de Michigan, 1995, p. 392-514. Rebeca SAN MARTín BASTIDA, «De Dalí a Lorca: El poema en prosa surrealista ", forma breve 2, 2004, p. 81-103.

16. L'Amic de les Arts, Sitges, juliol 1927. Lluis Montanya, Sebastià Gasch et Salvador Dalí, auteurs du « Manifest groc » en 1928 associèrent Lorca à leurs activités dans les milieux d'avant-garde catalans.

17. « He recibido L'amic de les Arts y he visto el prodigioso poema de tu hermano. Aquí en Granada lo hemos traducido y ha causado una impresión extraordinaria, «A A. M ${ }^{a}$ Dalí, Granada agosto 1927 », O.C. VII, Op. cit., p. 983.

18. Poeta en Nueva York. Primera edición del original fijada y anotada por Andrew A. Anderson, Barcelona, Galaxia Gutenberg, 2015, p. 240-242. Auparavant, M. García-Posada avait fait le choix de l'exclure dans son édition des œuvres complètes chez Galaxia Gutenberg, rééditées en poche chez Akal.

19. Domingo RóDENAS donne trois explications à ce phénomène, dans la lignée des postures de la Revista de Occidente: une certaine défiance vis-à-vis des vers, option facile et artificielle pour certains ; un besoin d'équilibrer les contributions en vers et en prose dans les publications; la volonté commune aux acteurs de forger une prose moderne, Prosas..., Op. cit., p. 28-29.

20. « A G. de Torre, Granada febrero 1927 », O. C. VII, Op. cit., p. 946 et 947.

21. « A S. Gasch, Lanjarón, 25 agosto 1927 », Ibid., p. 987.

22. « A S. Gasch, Madrid, 24 noviembre 1927 », Ibid., p. 997.

23. « A M. Fernández Almagro, Granada Cadaqués, julio 1927 », Ibid., p. 977.

24. «A S. Gasch, Granada septiembre 1927 », Ibid., p. 993.

25. « A R. Guillén, Granada enero 1927 », Ibid., p. 929.

26. «Todo el día tengo una actividad poética de fábrica », «A Jorge Zalamea, Granada septiembre $1928 »$, Ibid., p. 1030.

27. « Poética », O. C. VII, Op. cit., p. 477.

28. M. G. Posada parle de « Lorca como el poeta quizá más culto de su generación, el trabajador, el unificador de la tradición y de las vanguardias en una síntesis que no dio escuela », O. C. I, Op.cit., p. 32. Voir aussi Luis GARCÍA MONTERo, Un lector llamado Federico García Lorca, Madrid, Taurus, 2016.

29. « A M. Fernández Almagro, Granada enero de 1927 », O. C. VII, Op. cit., p. 932.

30. Ibid., « A J. Zalamea, Granada septiembre 1928 », p. 1029-1030.

31. Ibid., « A S. Gasch, Granada 8 septiembre 1928 », p. 1034. 
32. Ibid., « A S. Gasch, Granada septiembre 1928 », p. 1035.

33. Salvador DALÍ, « Sant Sebastià », L'Amic de les Arts, any II, 16, 31 juliol 1927, p. 52-54.

34. « Meditaciones y alegorías del agua », Pez..., Op. cit., p. 85.

35. «Corazón bleu y cœur azul », Poemas..., Op. cit., p. 91.

36. Pez..., Op. cit., p. 14.

37. Sur ce point, voir Zoraida CARANDELL, Lecture de Poeta en Nueva York. García Lorca ou la déréliction lyrique, Rennes, Presses Universitaires de Rennes, à paraître en septembre 2020.

38. « Telegráfo », op.cit., p. 617.

39. Ibid., p. 613-614.

40. Pez..., Op. cit., p. 97. Cette figure du jardinier est l'écho de la fin de «Sombras », sous-titré " poema en prosa ", un dialogue d'âmes en cours de disparition, qui laissent place au jardinier et à son fils sur le point de planter trois arbres, Teatro inédito de juventud, Madrid, Cátedra, Andrés Soria Olmedo (ed.), 1994, p. 317.

41. « Meditaciones y alegorías del agua », Pez..., Op. cit., p. 85-86.

42. "Juego de damas ", Ibid., p. 82.

43. «Meditaciones y alegorías del agua », Ibid., p. 87.

44. « Paseo », Poesía completa..., Op. cit., p. 616.

45. « Santa Lucía y San Lázaro ", Pez..., Op. cit., p. 43.

46. Je suis en cela d'accord avec ce qu'affirme E. ALONSO VALERO. Si la destruction de « lo viejo » est une constante, il est plus juste de parler de transformation que de rupture dans le cas de Lorca: «En realidad, sobre todo en el caso de García Lorca, tendríamos que hablar siempre de redefinición de unas categorías a partir de otras, de discontinuidades y retornos, no de ruptura ni de renuncia total ", No preguntarme nada. Variaciones sobre tema lorquiano, Granada, La razón poética, Atrio ensayo, 2005, p. 11.

47. Antonio MONEGAL, «Bajo el signo de la sangre (algunos poemas en prosa de F. García Lorca) », Bazar, 4, p. 66.

48. «La representación de la violencia tiene también una lectura que no es literal, sino como metáfora de la violencia que se ejerce contra el discurso. Lo poético no es la mutilación misma, sino el tipo de recombinación y yuxtaposición de los signos que permite ", Antonio MONEGAL, En los límites de la diferencia. Poesía e imagen en las vanguardias hispánicas, Madrid, Tecnos, 1998, p. 102.

49. Quel exemple plus parlant de ce processus paradoxal que La Disparition [1968] de Georges Pérec, où le E/eux disparu(s) rend plus que présents les absents.

50. Poemas..., Op. cit., p. 60.

51. Ibid., p. 103.

52. Le mot est ici utilisé à dessein car il exhibe une réalité, et parce qu'il a nourri les jeux littéraires sérieux de Lorca, Buñuel et Dalí dans leur persécution des médiocres et conventionnels, les «putrefactos ». Voir Salvador DALí, F. GARCíA LORCA, Los putrefactos. Dibujos y documentos, Granada, Ayuntamiento de Granada, 1995.

53. Peter BROWN, Le culte des saints. Son essor et sa fonction dans la chrétienté latine [1981], trad. d'Anne Rousselle, Paris, Editions du CERF, CNRS, 2012.

54. «San Miguel. Granada », Poesía completa..., Op. cit., p. 380.

55. «San Rafael. Córdoba », Ibid., p. 382-383.

56. « Mártir de Santa Olalla », Ibid., p. 400.

57. P. BROWN, Le culte..., Op. cit., p. 67.

58. «DI », Poemas..., Op. cit., p. 81.

59. « DB », Ibid., p. 79.

60. «SS », Ibid., p. 65.

61. «Ella demostró en la plaza pública, ante el asombro del pueblo, que mil hombres y cincuenta pares de bueyes no pueden con la palomilla luminosa del Espíritu Santo. Su cuerpo, su cuerpazo, 
se puso de plomo comprimido. Nuestro Señor, seguramente, estaba sentado con cetro y corona sobre su cintura », Ibid., p. 59.

62. Ibid., p. 63.

63. « NS », Pez..., Op. cit., p. 46.

64. «SA », Ibid., p. 49 et 50.

65. « DI », Ibid., p. 59.

66. «La disparition comme faveur, chant de l'éloignement dans le paysage antérieur », Cees NOотЕвоOM, Autoportrait d'un autre [1993], Paris, Actes sud, 1994, p. 64.

67. Je nomme ainsi par un néologisme les aphorismes métapoétiques composés par Lorca dans un dialogue avec Dalí à partir de la pratique du collage, réunis dans « Consejos a un poeta ».

68. «Consejos a un poeta », O. C. VI, Op. cit., p. 294.

69. Dans Lysis (385-380 av. J. -C.), Socrate dialogue avec des jeunes hommes dans un gymnase d'Athènes, espace de contact homophile : l'un d'entre eux, Ctésippe, s'amuse de l'amour enfiévré que porte Hippotalès à Lysis, jeune garçon plein de grâces physiques et morales. Cependant, ce dialogue, s'il a pour objectif de renverser les opinions communes sur l'amitié, interroge principalement l'amour et son expression, avant de poser de nombreuses questions sur l'amitié. Il fait partie de ces dialogues dits «aporétiques » qui n'offrent ni définition ni réponse: Platon ouvre de profonds questionnements quant au désir qui se trouve au cœur de toute relation humaine. L'ambiguïté entre Eros et Philia, l'absence de différenciation nette entre relation amicale et relation amoureuse, entre fraternité et homoérotisme, permettent de lire le Lysis comme un discours sur le désir homosexuel.

70. « Árbol de sorpresas », Pez..., Op. cit., p. 95-96.

\section{RÉSUMÉS}

Les poèmes en prose et proses poétiques de Lorca forment un corpus singulier: des textes de jeunesse à ceux d'avant-garde, ils sont tous un creuset d'expérimentation constitutifs d'un véritable projet poétique, et ne constituent pas en cela des essais ou brouillons négligeables. Leur étude permet de réévaluer l'unité de la recherche en prose de Lorca au sein d'une vision palimpseste de la créativité. En eux, il élabore une poétique du frémissement, du tremblement puis du saisissement et un dire autre qui est aussi fondamentalement celui du poème en prose. Le protéisme formel et le rejet des liens esthétiques s'ancrent dans une posture critique et ironique qui est celle de la reprise. Le dépassement des limites formelles et génériques est un acte de liberté et d'émancipation qui se construit sur la disparition: fulgurances, évanouissements, métamorphoses, morts violentes, décompositions, ces figures peuplent la prose vers laquelle Lorca se déporte dans sa recherche lyrique.

Lorca's prose poems and poetic prose form a unique corpus: from early texts to avant-garde ones, all are a crucible for experimentation amounting to a genuine poetic project, far more than a mere set of essays or drafts. Studying them leads us to reassess the unity of Lorca's prose research within a palimpsestic view of creativity. In them, he develops a poetics that quivers, trembles then arrests, in a new expressive form which is also fundamentally that of the prose poem. Proteism in form and rejection of aesthetic ties are rooted in an ironic critical posture that involves reworking. Overstepping formal and generic limits is an act of freedom and emancipation built on disappearance: lightning flashes, fainting away, metamorphoses, violent 
deaths, decomposition, such figures populate the prose that Lorca is moving towards in his lyrical research.

INDEX

Mots-clés : poème en prose, prose poétique, Lorca, expérimentation, avant-gardes, surréalisme Keywords : prose poem, poetic prose, Lorca, experimentation, avant-garde, surrealism

\section{AUTEUR}

CAROLE FILLIÈRE

(Université de Toulouse Jean Jaurès) 